\title{
Upcoming Physics Challenges for Device Modeling
}

\author{
R.Brunetti* and E. Piccinini ${ }^{\dagger}$ \\ ${ }^{*}$ CNR-INFM National Research Center for Nanostructures and Biosystems \\ at Surfaces (S3) and Physics Department \\ University of Modena and Reggio Emilia, Via Campi 213/A, 41100 Modena, Italy \\ brunetti.rossella@unimore.it \\ ${ }^{\dagger}$ CNR-INFM National Research Center for Nanostructures and Biosystems \\ at Surfaces (S3) and \\ Department of Electronics, Computer Science and Systems (DEIS) \\ University of Bologna, Viale Risorgimento 2, 40136 Bologna, Italy
}

\begin{abstract}
Science and technology at the nanoscale size offer today fundamental challenges in the field of device modeling. In this paper we document the growing interest of academies, institutions, and industries and the present impact on the market, and discuss different design strategies that have been proposed and/or implemented, aimed at the practical realization of innovative nanodevices. Few main examples of ideas and devices, namely ion-channel nano-biosensors, biomimetic sensors, molecular devices, solid-state components for quantum computation, nanotube electronics, and non-volatile nanomemories, are also revised.
\end{abstract}

\section{Making it small makes the difference}

There is a large consensus among scientists about the fact that when something (material, device, system or process) is developed on a truly nanometric scale (1-10 nm) its properties and behavior become different from those observed on the sub-micron mesoscale (10-100 nm). For this reason nanoscience and nanotechnology nowadays offer challenging problems from the point of view of fundamental research, and new fields for innovative industrial applications, both supported by many major funding initiatives worldwide. Many scientists, mainly physicists, chemists, and materials and electronics engineers have explored so far the new opportunities offered by nano- science and technology. Furthermore, since the typical space and time scales involved in nanoscience are in common with molecular biology, an impressive merging of knowledge is taking place, and collaborations are being established with the molecular-biology community. Two different approaches can be identified within this melting crucible. The engineer's approach is aimed at designing nanoscale elements and devices that should mimic, or even exceed, the performances of biological structures by making rational choices of materials, rather than accepting those that evolution has provided, and by using the modern principles of engineering design. The biologist's approach, based on the concept that the biological design solutions are likely to be close to the optimum for the environment in which they have evolved, is focused on removing the components of 
living systems from their biological homes and putting them to work in hybrid environments.

Although the progression of the semiconductor science and technology from "micro" to "nano" seems to be a natural evolution imposed by the miniaturization requirements, it is far from true that everything works in the same way, or even better, as soon as we are able to build it on the truly nanoscale size. Getting closer to the atomic scale introduces effects that require different design strategies. Moreover, complications in the deviceenvironment interaction may impose strong changes in the device and process design developed in the last twenty years. Thus "From lab to the market" is a long way: at present there are many promising effects, some engineered prototypes, and a relatively low number of industrial applications. The aim of this paper is to give an overview on some innovative ideas of truly-nano devices and sensors, and stress why they can be really innovative with respect to conventional products of the semiconductor industry. Sect. 2 provides some general information about the fields of research involved in the "nanorace" and the amount of investments worldwide. Examples relevant for the scope of the SISPAD conference are illustrated in Sect. 3.

\section{Is there really plenty of space at the bottom?}

Since the debut of the Scanning Tunneling Microscope (STM) in 1981, followed by the family of Atomic-Force Microscopes (AFM) few years later, which opened the doors to the nanoworld (see, e.g., [1] and references therein), nanoscience and nanotechnology collected the efforts of scientists, researchers, business managers, investors, funding agencies and governments worldwide. Around 50,000 people and 484 companies attended the 2007 International Nanotechnology Exhibition in Tokyo. In 2005 over 300 nanotechnology-based products have entered the marketplace with an estimated budget of over \$ 32 billion [2]. Scientific publications in the last years (from 1999 to 2004, more than 20,000 in the EU, more than 18,000 in the US, approximately 8,000 in Japan and 7,000 in China [3]) and the number of scientific journals specifically focused on nanotopics (more than 30) provide a good estimate of the fundamental and applied scientific activity of the last years. The huge estimated funding for nanoscience and nanotechnology worldwide (about 3.0, 2.2 and 2.0 billion of Euros in the US, Japan and EU, respectively [3]) indicates that the global expectation of the society about the nano research in the technologically advanced countries is very high.

However, the total number of patented applications in nanotechnology (growing from about 1,000 in 1995 to more than 2,500 in 2003 [3]), compared to the huge amount of basic and applied research efforts, seems to suggest that "the emergence of atomic-scale manufacturing on an industrial scale is still some decades away" [4].

It is hard to foresee when and to which extent many of the industrial sectors will be transformed by nanoscience and nanotechnology, despite the optimistic view of some nanoprophets. On the other hand, it is true that the scientific and technological progresses in the exploration of the nanoworld are now bringing the most appealing physics challenges to device modeling, significant benefits to many engineering disciplines, and innovation to manufacturers. 


\section{Innovative ideas for devices and sensors: examples from the nano- crucible}

\subsection{Ion-channel nano-biosensors}

The progress in X-ray structural-analysis techniques and sample preparation makes it now possible to investigate cells, and even part of them, on the atomic scale. One of the most outstanding results of these researches was the identification of ion channels, i.e., proteins forming nanometric pores across the cell membranes and ruling in- and outcell matter transport. Common general properties of ion channels include fast gating mechanisms and a high selectivity, that acts as a molecular sieve [5]. By combining and engineering these two properties, molecular devices able to switch on or off upon request in a very controlled way, and sensors with enhanced sensitivity, can be designed and built.

Computational tools based on classical and quantum mechanics, like Molecular Dynamics, Brownian Dynamics, and Monte Carlo algorithms, are mainly used to investigate ion-permeation features. The simulation of a channel protein embedded into a given membrane requires to include in the simulation up to many tens or a few hundreds of thousands atoms, which, in turn, implies big efforts in terms of computational resources.

Gramicidin A, porins, and potassium channels are the most studied protein channels. Theoretical models for the conduction mechanisms [6], selectivity [7], and current and noise properties [8] are available and provide results in good agreement with experiments. These results suggest that the innovation introduced by ion channels functionalized as biosensors would be the enhanced control of the analyte, i.e., of the substance to be detected, which can be in principle revealed even at molecular concentration. In fact, due to their conformation, ion channels filter and let ions (or clusters of few ions) pass through the membrane one at a time, in a single-file. If, after flowing through the channel, ions are collected into an engineered binding site fully integrated into a conventional circuitry, (almost) discrete current steps are recorded. The frequency of occurrences can in fact reveal the concentration of the analyte.

The current technological limiting factor to the application of these molecules as sensors is the insertion of the proteins into an artificial membrane and the integration with microfluidics and on-chip electronics, without perturbing the biological key-features of the proteins themselves. So far, gramicidin A proteins embedded in artificial polymeric membranes have been used to create a device integrated on a Si-based chip, preserving the "in vivo" functionality of the protein [9].

\subsection{Biomimetic sensors}

Traditional "electronic-noses", i.e., analyte-specific sensors, are usually composed by an array of sensors, each of them carrying a slightly different, some square micronswide membrane as sensing element. A particle of the substance to be detected is thus trapped inside the membrane by diffusion or ad-sorption, giving origin to a gravimetric, calorimetric or electromagnetic signal, which is, in turn, transformed into a current signal (see [10], [11], [12] and references therein). In order to reproduce or improve 
these functionalities at the nanoscale, a completely new design of the whole sensor must be achieved.

The exploitation of biological features at the nanoscale suggests designs for possible biomimetic sensors where the sensing function is performed by receptors instead of artificial membranes. These sensors temporarily trap molecules of the analyte in specific binding sites, like it happens in mammals' or insects' sensing system [13]. In biomimetic sensors, nanoelectronics and biological features merge together: proteins or organic supra-molecular complexes are placed on a semiconductor substrate, which includes electrodes and circuitry, preserving their capability to establish temporary weak interactions (Van der Waals, dipole/dipole, H-bonds, $\pi$ - $\pi$ interactions) with specific analytes. The protein backbone can also form steric constraints with the trapped molecules. Following such a scheme, once a molecule is grabbed by or bonded to the protein, a reaction takes place between the analyte itself and the protein, so that information is somehow transduced from the analyte-protein complex to the circuitry. After that, the grabbed particle is chemically inactivated by the system itself and released from the binding site. A new detection is then possible.

The sensing strategy described above implies an appropriate preliminary analysis of the sensing element in terms of supra-molecular reception. This requires an ad-sorption and catalysis-inspired design, a suitable thermodynamic knowledge of the protein folding and geometry, and of the reactions involving the analyte-protein complex. In some cases a spectrometric analysis may provide further information. Moreover, analyte-specific side groups can be added to the protein backbone through modern protein-manipulation techniques aimed at enhancing the sensor performances. The self-assembly capability of biological entities can also be exploited. The design and optimization of biomimetic sensors are thus very complex activities, that make use of the whole set of numerical tools cited in subsection 3.1, and imply a synergy among complementary branches of the scientific community.

At present, few prototypes of biomimetic sensors have been developed [13], [14]. If compared to standard sensors, they show an improved intrinsic selectivity and an enhanced sensitivity. Surface design and tailored proteins both represent future perspectives.

\subsection{Molecular devices}

The idea of using molecular building blocks for the fabrication of both passive (e.g., resistive wires) and active (e.g., transistors) devices dates back to the 1940ies, when the first study of charge and energy transfer in molecules was presented [15]. Molecular electronics (ME), thanks to the size reduction offered by molecular-level control of the electrical properties, in principle opens the way to extend Moore's Law beyond the foreseen limits of small-scale conventional silicon integrated circuits.

The direct measurement of the electronic characteristics of individual molecules awaited the development of methods for fabricating molecular-scale electrical contacts. Thus, the first experiment measuring the conductance of a single molecule was reported only in 1997 [16]. Since then, this branch of the field has progressed rapidly. Likewise, as it became possible to directly measure such properties, in many cases the theoretical predictions of early workers have been confirmed. Since the 1970ies, scientists have 
developed an entire panoply of new materials and devices.

Rapid progresses in nanotechnology and nanoscience provided new tools for the experimental and the theoretical study of molecular electronics. In particular, the use of STM and AFM facilitated the deposition and manipulation of single molecules for ME purposes. Furthermore, ME opened doors to plastic electronics and optoelectronics, which now begin to find commercial applications, though concerns still exist about the stability of the organic molecules in an electronic device.

From the modeling side, the knowledge of the transport mechanisms in molecular devices is still an open question, due to the subtle influence that many so-called "side effects" (usually neglected on a wider scale) have on single-molecule systems. Thus, it is likely to suppose that modeling molecular devices requires a new simulation approach able to properly treat the quantum-mechanical aspects of these nano-systems.

Molecular systems composed by a relatively large number of atoms can be studied nowadays by means of quantum-mechanical methods. An example of is offered by the $g D F T B$ method, which combines the well-known density-functional tight-binding (DFTB) method with the non-equilibrium Green-function approach [17]. By means of this computational tool, the tunneling current flowing across the organic link between contacts and the charge-density distribution within the device, can be computed consistently with the non-equilibrium boundary conditions imposed on the two contacts in presence of an applied voltage. Moreover, this approach accounts for the computation of the atomic forces acting on each atom, allowing for Molecular-Dynamics simulations that can be coupled to real-time current computations.

Case studies aimed at the self-consistent computation of I-V characteristics, charge densities and potentials of molecular systems bridging metal contacts have been tackled so far by the $g D F T B$ method, including the benzene-dithiol system, alkanethiols, and Tour wires [17]. From this analysis it emerges that the conduction mechanism in nano-devices is ruled by quantized currents, depending on the number of conducting channels. Moreover, since the overall properties of the device strongly depend on the characteristics of the contacts, it is impossible to define the conductivity of a device composed by one or few molecules.

\subsection{Solid-state nanocomponents for quantum computation}

Since the last decades quantum-information theory has been rapidly developed on the basis of the new possibilities offered by merging quantum mechanics principles and nanotechnology. However it is still very difficult to find a quantum system suitable for a technologically-feasible realization of the fundamental logic gates and easily integrable into a traditional circuity.

As in classical information theory, two-state systems (qubits) are required to encode and process information, and quantum mechanics principles are used to implement logic gates. It was proved that any logic gate can be realized by a sequence of one qubit rotation and two-qubit CNOT gates [18].

An example of a solid-state semiconductor system that can be designed as a qubit is offered by coherent electron transport in two identical parallel semiconductor quantum wires separated by a potential barrier of controlled height [19]. When an electron is injected into one of the two wires, it is possible either to transfer the electron wave 
function from one wire to the other (NOT gate) or to split the wave function between the two wires, by tuning the electron velocity and the potential barrier between the wires in a coupling region of finite length. The CNOT gate is implemented by adding a second pair of quantum wires, or control qubit, to the NOT gate (data qubit), in such a way that the Coulomb interaction between the data and control qubit is significant only if the electron in the control qubit resides in the appropriate wire of the couple, being negligible when it is in the other wire. As a result, depending on the position of the electron in the control qubit, the electron in the data qubit can realize a NOT gate or can be slowed down by the Coulomb interaction between the two wires, ending up in the original wire without changing its logical state.

The properties of qubits are theoretically investigated by means of a numerical solution of the time-dependent two-dimensional Schrödinger equation [19]. Furthermore, it has been proved that Surface Acoustic Waves improve the functionality of the qubits by preventing the spreading of the electron wave function and reducing undesired reflection effects [20].

Coherent coupling of vertical [21] or lateral [22] double quantum wires has been recently proved. Experimental results indicate that the current in a wire is influenced by the inter-wire coupling strength. At present, the implementation of double quantum wires for CNOT gates still represents a challenge of modern semiconductor nanotechnology.

\subsection{Nanotube electronics}

Single-walled carbon nanotubes (SWNTs) exhibit interesting transport properties at room temperature, like, e.g., mobilities more than 10 times higher than Si, current per unit areas as high as $10^{9} \mathrm{~A} \mathrm{~cm}^{-2}$, and ideal transistor subthreshold characteristics. However the use of single SWNTs as functional elements cannot be considered, due to both their low current outputs and small active areas. In addition, there are also serious technological problems about synthesizing large numbers of perfectly aligned, linear, individual, electrically homogeneous tubes, and accurately positioning them to form a regular array.

Recently, the problems cited above have partially been solved: arrays of individual SWNTs with average diameters of $1 \mathrm{~nm}$, lengths up to $300 \mu \mathrm{m}$ and densities approaching $10 \mathrm{SWNTs} / \mu \mathrm{m}^{2}$ have successfully been deposited on a quartz wafer [23] using chemical vapor deposition, with perfectly linear configurations within the measurement resolution of AFM. In order to integrate these arrays into an isolated transistor, source and drain electrodes ( $1 \mathrm{~nm}$ Ti or 20nm Pd) are deposited through photo-lithography on the SWNTs/quartz substrates. The SWNTs outside the channel region are etched, a uniform epoxy gate dielectric is deposited on the top structure and, finally, top-gate electrodes $(1 \mathrm{~nm} \mathrm{Ti}$, or $20 \mathrm{~nm} \mathrm{Au})$ aligned to the channel region are created using photolithography.

These devices exhibit extremely high current outputs, high on-off current ratios, good transconductances, and can be placed on rigid or flexible substrates. These results indicate a scalable path to SWNTs-based thin-film electronics with high-performance capabilities. Initial applications can be envisioned in areas that require unusual substrates (e.g., plastics). The possibility of direct integration with Si also creates interest in the 
possible use of nanotube-array devices with Si CMOS for enhanced operation.

\subsection{A non-volatile memory at the nanoscale}

Programmable Metalization Cell (PMC) memory [24] is an example of a nanodevice already available on the market. Its design and working principle rely on the electrochemical control of nanoscale quantities of metal in thin films of solid electrolyte. In solid electrolytes containing metal ions, only the positively-charged ions are mobile, while the negatively-charged counter-ions are fixed in the solid. The device is formed by an anode containing an oxidizable metal (e.g., $\mathrm{Ag}$ or $\mathrm{Cu}$ ) and an electrochemically inert cathode (e.g., $\mathrm{W}$ or $\mathrm{Ni}$ ), which are formed in contact with a layer of solid electrolyte. Silver or copper can be added to a variety of glasses to form solid electrolytes. Base glasses can be chosen among a variety of chalcogenides (e.g., compounds of S or Se) or oxides.

If an external voltage as low as a few hundred $\mathrm{mV}$ is applied, ions are reduced to form metal atoms. The electrodeposition of metal on the cathode physically extends the electrode into the electrolyte. This physical intrusion is possible in solid electrolytes, particularly if they are glassy in nature and can accommodate the growing electrodeposit in their void-rich, semi-flexible structure. Since the electrodeposit is connected to the cathode, it can supply electrons for subsequent ion reduction. So the advancing electrodeposit will harvest ions from the electrolyte, plating them onto its surface to extend itself forward. The average distance each ion has to travel in order to be reduced is a few $\mathrm{nm}$ at most. The resistivity of the electrodeposit is many orders of magnitude lower than that of the surrounding electrolyte.

Thus, once the electrodeposit has grown from the cathode to the anode (typically separated by a distance of $50 \mathrm{~nm}$ ), the resistance of the structure drops considerably and the current flowing through the device increases until the current limit imposed by the source resistance is reached. At this point the voltage drop falls to the threshold for electrodeposition and the process stops, yielding the final on-resistance of the structure. The electrodeposition is reversible by changing the polarity of the applied bias, and much the same rules that govern growth apply. The device also shows excellent endurance with no significant degradation to over $10^{10}$ cycles.

\section{Acknowledgments}

We are very grateful to A. Di Carlo, S.M. Goodnick, and D. Narducci for providing information and data from their own research. We also thank A. Bertoni, P. Bordone, C. Jacoboni, and M. Rudan for useful discussions and suggestions.

\section{References}

[1] C. Gerber and H.P. Lang, "How the doors of the nanoworld opened", Nature Nanotechnology, vol. 1, pp. 3-5, 2006.

[2] S.C. Curral, E.B. King, N. Lane, J. Madera and S. Turner, "What drives public acceptance of nanotechnology?", Nature Nanotechnology, vol. 1, pp. 153-155, 2006.

[3] A. Hullmann, "Who is winning the nanorace?", Nature Nanotechnology, vol. 1, pp. 81-83, 2006. 
[4] T. Theis in “nan'o.tech.nol'o.gy n.", Nature Nanotechnology, vol. 1, pp. 8-9, 2006.

[5] B. Hille, "Ionic Channels of Excitable Membranes", 3rd Ed., Sinauer, Sunderland, MA, ISB N: 0-878933-21-2, 2001.

[6] S. Bernèche and B. Roux, "Energetics of ion conduction through the K+ channel", Nature, vol. 414, pp. 73-77, 2001.

[7] M. LeMasurier, L. Heginbotham and C. Miller, “KcsA: It's a Potassium Channel”, Journal of General Physiology, vol. 118, pp. 303-313, 2001.

[8] E. Piccinini, F. Affinito, R. Brunetti, C. Jacoboni and M. Rudan, "Computational Analysis of Current and Noise Properties of a Single Open Ion Channel", Journal of Chemical Theory and Computation, vol. 3, pp. 248-255, 2007.

[9] S.J. Wilk, L. Petrossian, M. Goryll, G.M. Laws, S.M. Goodnick, T.J. Thornton, M. Saraniti, J. Tang and R.S. Eisenberg, "Ion Channels on Silicon", e-Journal of Surface Science and Nanotechnology, vol. 3, pp. 184-189, 2005.

[10] J.W. Grate, S.J. Martin and R.M. White, "Acoustic wave microsensors", Analytical Chemistry, vol. 65, pp. 940A-948A, 1993 and Analytical Chemistry, vol. 65, pp. 987A-996A, 1993.

[11] K.D. Schierbaum, A. Gerlach, M. Haug and W. Göpel, "Selective detection of organic molecules with polymers and supramolecular compounds: application of capacitance, quartz microbalance and calorimetric transducers", Sensors and Actuators A, vol. 31, pp. 130-137, 1992.

[12] H. Baltes, D. Lance and A. Koll, "The electronic nose in Lilliput", IEEE Spectrum, vol. 35, pp. 35-38, 1998

[13] K. Toko, "A taste supersensor", Measurement Science and Technology, vol. 9, pp. 19191936, 1998.

[14] D. Narducci, Patent \# ITRM2005A000092.

[15] N.S. Hush, "An Overview of the First Half-Century of Molecular Electronics", Molecular Electronics III, vol. 1006, pp. 1-20, 2003.

[16] M.A. Reed, C. Zhou, C.J. Muller, T.P. Burgin, J.M. Tour, "Conductance of a Molecular Junction", Science, vol. 278, pp. 252-254, 1997.

[17] A. Pecchia, L. Latessa, A. Gagliardi, T. Frauenheim and A. Di Carlo "The gDFTB Tool for Molecular Electronics" in "Molecular Electronics: Analysis, Design and Simulation", J.M. Seminario Ed., Elsevier, Amsterdam, The Netherlands, ISB N: 0-444-52725-7, 2006.

[18] T. Sleator and H. Weinfurter, "Realizable Universal Quantum Logic Gates", Physical Review Letters, vol. 74, pp. 4087-4090, 1995.

[19] A. Bertoni, P. Bordone, R. Brunetti, C. Jacoboni and S. Reggiani, "Quantum Logic Gates based on Coherent Electron Transport in Quantum Wires", Physical Review Letters, vol. 84, pp. 5912-5915, 2000.

[20] P. Bordone, A. Bertoni, M. Rosini, S. Reggiani and C. Jacoboni, "Coherent transport in coupled quantum wires assisted by surface acoustic waves", Semiconductor Science and Technology, vol. 19, pp. S412-S414, 2004.

[21] S.F. Fischer, G. Apetrii, U. Kunze, D. Schuh and G. Abstreier, "Energy spectroscopy of controlled coupled quantum-wire states", Nature Physics, vol. 2, pp. 91-96, 2006.

[22] A. Ramamoorthy, J.P. Bird and J.L. Leno, "Switching characteristics of coupled quantum wires with tunable coupling strength", Applied Physics Letters, vol. 89, pp. 0131181-013118-3, 2006.

[23] S. Jun Kang, C. Kocabas, T. Ozel, M. Shim, N. Pimparkar, M.A. Alam, S.V. Rotkin and J.A. Rogers, "High-performance electronics using dense, perfectly aligned arrays of carbon nanotubes", Nature Nanotechnology. vol. 2, pp. 230-236, 2007.

[24] M.N. Kozicki, "Highly scalable resistance-change memory using solid electrolytes", IEEE International Solid State Circuits Conference (ISSCC), San Francisco, CA, 2006. 